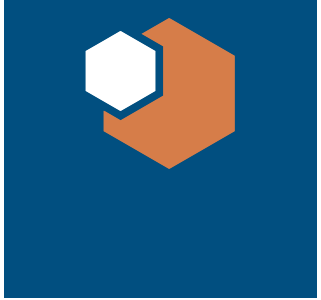

\section{Commercializing colloidal quantum dot photovoltaics}

\author{
By Ahmad R. Kirmani
}

$\mathrm{C}^{\mathrm{s}}$ olloidal quantum dots (CQDs) for next-generation photovoltaics (PV) and display technologies have been actively pursued over the last decade. Lead sulfide $(\mathrm{PbS})$ and, more recently, cesium lead triiodide $\left(\mathrm{CsPb}_{3}\right)$ perovskite CQDs have shown great promise as light absorbers. These are usually synthesized as colloids in organic solvents at a low cost. The ease of solution processability, strong light absorption, and size tunability of the bandgap have chiefly been behind the surge in research interest in this technology, especially due to a facile, hot-injection synthesis protocol for CQDs that Hines and Scholes ${ }^{1}$ developed in 2001. This protocol involves a rapid mixing of the reactants maintained at an elevated temperature, is low-cost and easy to perform at the laboratory scale, and yields high-quality CQDs.

Research on CQD solar cells has subsequently focused on perfecting defect-ridden CQD surfaces which, owing to a large surface-to-volume ratio, are home to a high density of trap states. These trap states slow down photo-generated charge carriers in CQD films used as absorber layers in solar cells, hurting the power-conversion efficiency (PCE) and, with it, the commercial prospects of this technology. Robust surface passivation schemes over the years, coupled with smart-device engineering, have taken PCEs from $<1 \%$ in 2005 to $>13 \%$

in 2018..$^{-4}$ It is believed that a further gain of a few PCE points might be sufficient to put CQD PV on the production line for standalone indoor applications and to complement existing commercial PV technologies, such as silicon.

The research group of Vladimir Bulović of the Massachusetts Institute of Technology finds that this approach of focusing solely on PCE might not be the most favorable route to commercialization of this technology. ${ }^{5}$ They found that the CQD synthesis step currently limits production costs. They also found that the currently used hot-injection synthesis method is significantly capital-intensive, owing to low yields and therefore incompatible with industrial scale-up.

A less-explored, diffusion-controlled heat-up method was found to lower the module cost. Synthesis of CQDs by the heat-up method involves mixing of the reactants prior to heating. ${ }^{6}$ The heat-up method allows for high yields resulting in reduced synthesis costs down to USD\$0.15/W from USD\$0.51/W for the hot-injection method (compared to $<\mathrm{USD} \$ 0.4 / \mathrm{W}$ for commercial silicon PV). ${ }^{5}$

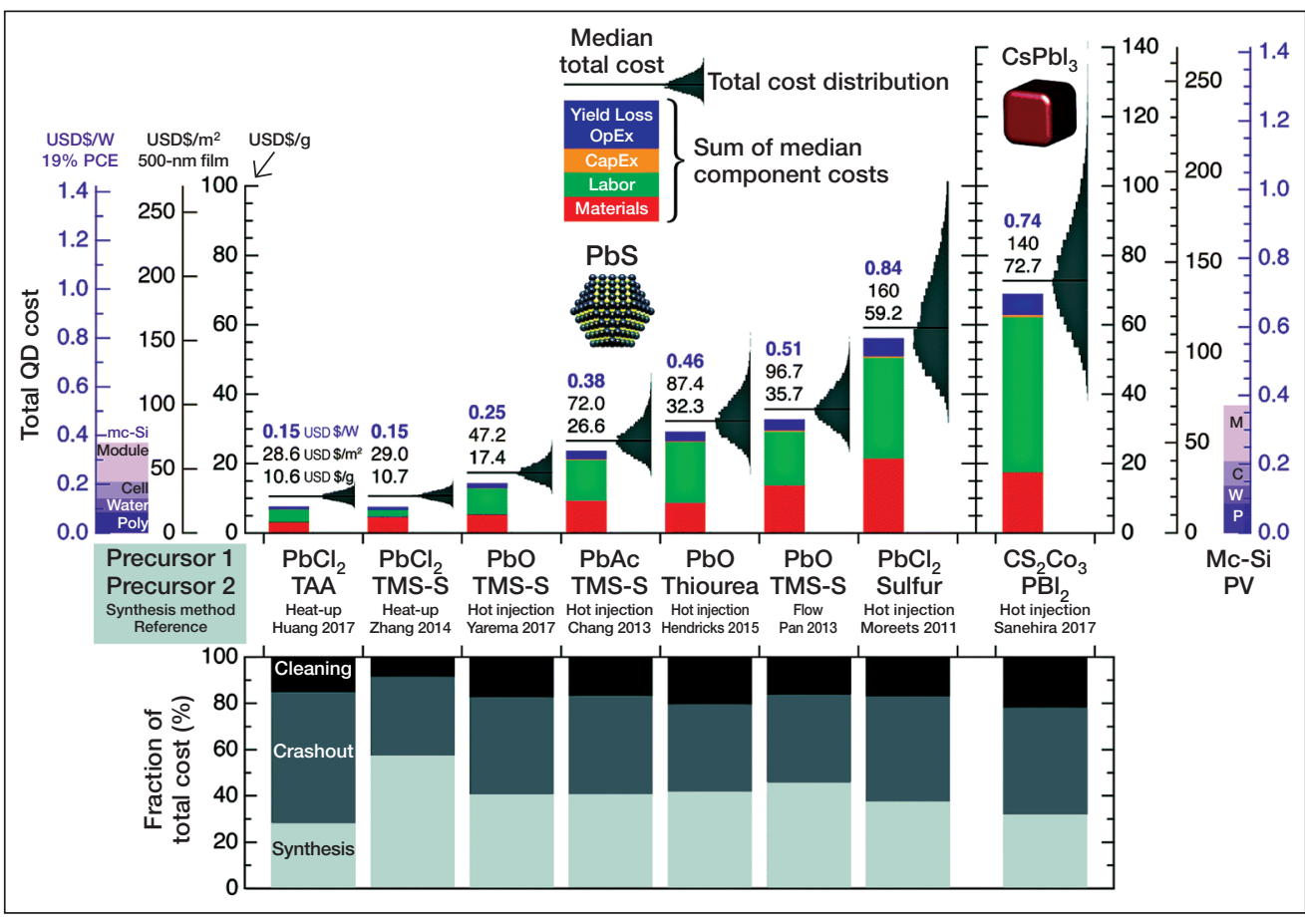

Figure 1. Results of the Monte Carlo cost model developed by the Bulović group summarizing the synthesis costs of $\mathrm{PbS}$ and $\mathrm{CsPbl}_{3}$ colloidal quantum dots (CQDs) by a variety of synthesis routes. ${ }^{5}$ Credit: Royal Society of Chemistry.
Additional encapsulant layers and balance-ofmodule components required for CQD PV, however, expectedly increase this number significantly. Jean et al. ${ }^{5}$ present guidelines to reduce CQD synthesis costs to as low as USD $\$ 0.05 / \mathrm{W}$, including avoiding expensive precursors, deploying lowergrade precursors, and recycling solvents. The article also discusses the commercial prospects of $\mathrm{CsPbI}_{3}$ CQDs, and the analysis suggests these to be significantly more expensive than PbS CQD PV.

"There is no fundamental reason why CQDs have to be expensive if the component materials are cheap, 
abundant, and produced in high volumes. If researchers can develop high-throughput automated synthesis techniques that use low-cost precursors, CQDs could still find widespread use in PV and many other commercial applications," says Joel Jean, the CEO of Swift Solar and first author of the study.

The field of PbS CQD PV has moved past solid-state ligand exchange (SSE), a necessary chemical route that was used to enhance charge transport in the CQD absorber layer, but required a layer-by-layer buildup of the absorber and was time- and materials-intensive. Recent demonstrations of carrying out this exchange in the solution phase have helped researchers realize high-performance solar cells fabricated in a single step using roll-to-roll compatible coating protocols such as blade coating. ${ }^{7,8}$ In their Monte Carlo cost model, Bulović's group considered a scalable and industry-compatible coating technique, slot-die coating, which is very similar in design and principle to blade coating. Although their model does not include spin coating - a standard laboratory-based coating technique - the process is unattractive for commercial purposes owing to $\sim 99 \%$ materials wastage. Blade coating, on the other hand, has been shown to achieve $0 \%$ materials wastage, allowing fabrication of $\sim 25$ times more devices for the same CQD volume as that used by spin-coated solar cells. ${ }^{8}$

This is not yet the case for $\mathrm{CsPbI}_{3}$ CQD solar cells, which currently do not have a similar robust solution-phase exchange (SPE) recipe; and rely on spincoating-based SSE. Although $\mathrm{CsPbI}_{3}$ CQDs hold great promise as a PV technology because of the extra benefits they bring, commercial prospects will depend on the success of fabricating devices using them through a low-cost route.

The analysis by Bulović's group considers strict environmental control for synthesis and device fabrication, as currently required, given the sensitivity of the fabrication process to ambient conditions. This view is expected to change with a recent report, where robust $\mathrm{PbS}$ CQD PV was shown to achieve long-term stability under high humidity. ${ }^{8}$ The study found that it is possible to fabricate these solar cells with negligible control over operational humidity, but an extra step of "oxygen doping" has to be performed to remove the ill effects of moisture. Reducing control over operational humidity is expected to reduce the overall synthesis and fabrication cost of CQD solar cells.

Another major recent development that is expected to bring down the cost is the demonstration of autonomous synthesis of CQDs. A research team led by Milad Abolhasani at North Carolina State University reported a technique called "QDExer" that automates the chemical synthesis step, producing high-quality CQDs. " "Continuous flow synthesis of CQDs using computer-controlled microreactors would require far less labor to synthesize industrially relevant quantities of high-quality CQDs. We estimate that a scaled-up microfluidic CQD synthesis platform could potentially reduce overall CQD manufacturing costs by at least 50 percent. By precisely mixing CQD synthesis reagents using a train of monodispersed microdroplets that flow through a microreactor, we can achieve superior mass transfer control compared to batch syntheses," Abolhasani says.

The rigorous model developed by the Bulović group presents guidelines toward commercialization of CQD PV. An exciting route forward is to synthesize electronically active and device-relevant CQDs with a high-throughput and cost-effective route, and QDExer technology could be the answer. SPE, coupled with a scalable fabrication protocol (blade, spray, slot-die, or inkjet), should be viable CQD film-printing techniques. Combined, these recent demonstrations potentially make CQD PV an industrial reality in the near future for indoor flexible PV solutions, and complement current commercial PV technologies in harnessing the IR spectrum of solar light that the latter cannot.

\section{References}

1. M.A. Hines, G.D. Scholes, Adv. Mater.15(21), 1844 (2003).

2. J. Xu, O. Voznyy, M. Liu, A.R. Kirmani, G. Walters, R. Munir, M. Abdelsamie, A.H. Proppe, A. Sarkar, F.P. García de Arquer, M. Wei, B. Sun, M. Liu, O. Ouellette, R. Quintero-Bermudez, J. Li, J. Fan, L. Quan, P. Todorovic, H. Tan, S. Hoogland, S.O. Kelley, M. Stefik, A. Amassian, E.H. Sargent, Nat. Nanotechnol. 13, 456 (2018).

3. S.A. McDonald, G. Konstantatos, S. Zhang, P.W. Cyr, E.J.D. Klem, L. Levina, E.H. Sargent, Nat. Mater. 4, 138 (2005).

4. E.M. Sanehira, A.R. Marshall, J.A. Christians, S.P. Harvey, P.N. Ciesielski, L.M. Wheeler, P. Schulz, L.Y. Lin, M.C. Beard, J.M. Luther, Sci. Adv 3 (10), eaa04204 (2017).

5. J. Jean, J. Xiao, R. Nick, N. Moody, M. Nasilowski, M. Bawendi, V. Bulović, Energy Environ. Sci. 11, 2295 (2018).

6. J. Zhang, J. Gao, E.M. Miller, J.M. Luther, M.C. Beard, ACS Nano 8 (1), 614 (2014).

7. M. Liu, O. Voznyy, R. Sabatini, F.P. García de Arquer, R. Munir, A.H. Balawi, X. Lan, F. Fan, G. Walters, A.R. Kirmani, S. Hoogland, F. Laquai, A. Amassian, E.H. Sargent, Nat. Mater. 16 (2), 258 (2017).

8. A.R. Kirmani, A.D. Sheikh, M.R. Niazi, M.A. Haque, M. Liu, F.P.G. de Arquer, J. Xu, B. Sun, O. Voznyy, N. Gasparini, D. Baran, T. Wu, E.H. Sargent, A. Amassian, Adv. Mater. 30 (35), 1801661 (2018).

9. K. Abdel-Latif, R.W. Epps, C.B. Kerr, C.M. Papa, F.N. Castellano, M. Abolhasani, Adv. Funct. Mater. (Suppl.), 1900712 (2019) 